\title{
Influence of Tonifying Kidney and Spleen, Detoxification of Throat Method to TNF- $\alpha$, IL-6 of IgA Nephropathy Model Rat
}

\author{
Di ZOU ${ }^{1, a}$, Yin-pin WANG ${ }^{2}$, Dong-mei ZHANG ${ }^{3}$ and Shou-lin \\ ZHANG ${ }^{4 b^{*}}$
}
${ }^{1,2,3,4}$ Department of Nephrology, the First Affiliated Hospital to Changchun University of Chinese Medicine, Changchun, Jilin province, China,130021.
aaggiezou@163.com, bshoulin-z@163.com
* Corresponding author

Keywords: Tonifying Kidney and Spleen, Detoxification of Throat Method, IgA nephropathy, Model rats, TNF- $\alpha$, IL-6.

\begin{abstract}
Purpose: To study the effect and function mechanism of Tonifying Kidney and Spleen, Detoxification of Throat method on IgA nephropathy. Methods: the 72 Wistar rats were randomly divided into blank group, model group, Lotensin group, Tonifying kidney and spleen, detoxification of throat high, medium and low dose group (which is called traditional Chinese medicine high, medium and low dose group for short). The IgA nephropathy rat model were established by the method of feeding with bovine serum albumin (BSA) and injecting tail vein with lipopolysaccharide (LPS).After Eight weeks administration, each phase's urine red blood cell count, $24 \mathrm{~h}$ UTP, TNF- $\alpha$ and IL- 6 content were observed. Results: compared with model group, every groups' UTP decreased and TCM medium dose group decreased extraordinary $(P<0.05)$;compared with model group, urine red blood cell count decreased significantly of every TCM group $(P<0.05)$, especially TCM medium dose group improved significantly $(P<0.01)$, but Lotensin group urine red blood cell count no drop $(P>0.05)$; compared with blank group, TNF- $\alpha$, IL-6 of model group's were significantly elevated $(P<0.05)$; compared with model group, TNF- $\alpha$,IL-6 of treatment groups decreased significantly $(P<0.05)$,compared between TCM group, TCM medium dose group's data is the most significant $(P<0.01)$.Conclusion: Tonifying kidney and spleen, detoxification of throat method can reduce hematuria and proteinuria of IgA nephropathy model rats, and reduce the content of IL- 6 and TNF- $\alpha$ in serum which is better than Lotensin.
\end{abstract}

\section{Introduction}

IgA nephropathy (IgAN) is a relatively common glomerular disease with high incidence in our country, accounting for about $30 \% \sim 40 \%$ of chronic nephritis. It is one of the leading cause of end-stage renal disease [1-2]. The pathological feature is that glomerular mesangial area appears diffuse IgA deposits with mesangial proliferation, its mainly clinical manifestation is hematuria and proteinuria. Tonifying kidney and spleen, detoxification of throat method was come up by national physician master Jixue Ren.It can effectively reduce the IgAN patient's hematuria and proteinuria, delay the deterioration of renal function. In order to further study the function mechanism of this method in the treatment of IgAN, the paper makes a research, the reported as follows. 


\section{Materials and Methods}

\section{Animals}

Choose 72 health and clean male Wistar rats in 6 weeks old, the weight is $160 \sim 200 \mathrm{~g}$, provided by animal laboratory, the Norman Bethune medical college of jilin university, experimental animals qualified number: SCXK (liao) 2013-000.

\section{Instruments Reagents and Drugs}

TXM-500C fluorescence microscope, provided by Shanghai Tiansheng instrument co.; Automatic enzyme standard meter 318C, provided by Shanghai Zuofei laboratory equipment co.; Organized crusher BIO - 950, provided by Shanghai Bannuo biological technology co.; TNF- $\alpha$ and IL-6 ELISA detection kits, provided by the Shanghai Xinyu biological technology co.; Bovine serum albumin and lipopolysaccharide, provided by America Sigma company; Fluorescein label rabbit sustain rat IgA antibodies, provided by Beijing Boorson biological technology co.; Lotensin provided by Beijing Novartis pharma co.(its national standard number: 1050423H0); Drugs towards Tonifying Kidney and Spleen, Detoxification of Throat method all supervised by the Affiliated Hospital to Changchun University of Chinese Medicine.

\section{The Method of Experiment}

Establish the Model. Firstly, feed the rats a week after purchase for letting them adapt the environment; secondly, randomly divided them into blank group, model group, Lotensin group, Tonifying kidney and spleen, detoxification of throat method high, medium and low dose group; thirdly, prepare ainimal model by referring to huiyu Liu[3] suggested method, and select 2 rats from each group in the ninth week; fourthly, check whether successfully establish the model by choosing nephridial tissue under microscope.

Grouping and Drug Delivery. After the successfully establish the model, the control group is given Lotensin $10 \mathrm{mg} /(\mathrm{kg} \cdot \mathrm{d})$ by gavage administration; the treatment group is given the dose of traditional Chinese medicine high, medium and low 32, 16 and $8 \mathrm{~g} /(\mathrm{kg} \cdot \mathrm{d})$ by gavage administration; the blank group and model group is only given saline lavage $4 \mathrm{ml}$ a day to the stomach. All of these continually to 8 weeks.

Observation. Observe the general condition of rats, including body quality, the hair color, the urine color and urine output, respectively at the weekend of $0,4,6,8$, $10,12,14$ collect the $24 \mathrm{~h}$ urine by metabolic cages. Then observe its color, record urine quantity, the frequency and degree of macroscopic haematuria, take part of urine to detect by UTP in $24 \mathrm{~h}$, check the count of urine sediment and urine red blood cell, and detect the content of TNF- $\alpha$ and IL- 6 in serum by ELISA method( operate under the guide of kit introduction)

\section{Statistical Methods}

Using SPSS 19 statistical software, measurement data was descripted with ( $\left.x_{ \pm \mathrm{s}}\right), P<$ 0.05 differences have statistical significance.

\section{Result}

\section{Common Situation}

There were 7 rats died during establish the model, and each group choose two rats are used to verify whether success. The blank group rats are always in good condition 
during the testing process. After the 4th week, rats eating and activity decreased, its hair became dark, the weight dropped, and appeared diarrhea, darker color of urine; Since the 10th week, with the drug treatment, the general condition of treatment group rats improved markedly and the weight increased.

\section{The Comparison of Urinary Red Blood Cell Count between Groups of Rats}

Compared with model group, urine red blood cell count decreased significantly in every traditional Chinese medicine group $(P<0.05)$, especially traditional Chinese medicine medium dose group improved significantly $(P<0.01)$, but Lotensin group urine red blood cell count no drop $(P>0.05)$; As shows in table 1 .

Table1.the comparison of urinary red blood cell count between groups of rats before and after treatment.(/HP)

\begin{tabular}{ccccccc}
\hline week & $\begin{array}{c}\text { The blank } \\
\text { group }\end{array}$ & $\begin{array}{c}\text { The model } \\
\text { group }\end{array}$ & $\begin{array}{c}\text { the Lotensin } \\
\text { group }\end{array}$ & $\begin{array}{c}\text { The high dose } \\
\text { group }\end{array}$ & $\begin{array}{c}\text { The med ium } \\
\text { dose group }\end{array}$ & $\begin{array}{c}\text { The low dose } \\
\text { group }\end{array}$ \\
\hline 0 & $4.82 \pm 0.52$ & $4.52 \pm 0.40$ & $3.24 \pm 0.33$ & $3.88 \pm 0.35$ & $4.09 \pm 0.63$ & $4.09 \pm 0.70$ \\
4 & $4.96 \pm 0.39$ & $5.30 \pm 0.93$ & $6.24 \pm 0.22$ & $5.23 \pm 0.63$ & $5.66 \pm 0.56$ & $5.49 \pm 0.42$ \\
6 & $4.77 \pm 0.53$ & $62.13 \pm 10.53^{\#}$ & $68.22 \pm 0.49^{\#}$ & $64.03 \pm 0.39^{\#}$ & $59.45 \pm 0.46^{\#}$ & $60.66 \pm 0.36^{\#}$ \\
8 & $4.42 \pm 0.34$ & $138.2 \pm 15.25^{\# \#}$ & $129.60 \pm 0.40^{\# \#}$ & $132.15 \pm 0.46^{\# \#}$ & $140.72 \pm 0.48^{\# \#}$ & $134.86 \pm 0.50^{\# \#}$ \\
10 & $4.21 \pm 1.21$ & $133.21 \pm 1.24$ & $132.79 \pm 1.72$ & $126.22 \pm 1.50$ & $129.08 \pm 1.56$ & $125.20 \pm 1.22$ \\
12 & $4.11 \pm 1.14$ & $161.43 \pm 1.83$ & $155.88 \pm 1.61$ & $118.17 \pm 1.28^{\triangle \Delta}$ & $108.56 \pm 1.45^{\triangle \Delta}$ & $120.97 \pm 1.60^{\triangle \Delta}$ \\
14 & $4.29 \pm 1.26$ & $172.81 \pm 0.60$ & $169.43 \pm 1.03$ & $99.73 \pm 0.58^{\Delta}$ & $87.63 \pm 1.01^{\Delta}$ & $95.85 \pm 1.15^{\triangle}$ \\
16 & $4.93 \pm 1.27$ & $166.49 \pm 1.80$ & $163.56 \pm 1.78$ & $88.33 \pm 1.20^{\triangle}$ & $79.56 \pm 0.58^{\triangle \Delta \bullet}$ & $84.90 \pm 1.40^{\triangle \Delta}$ \\
\hline
\end{tabular}

Note: compare with the blank group, \# $P<0.05$, \#\# $P<0.01$; compare with the model group ${ }^{\triangle} P<0.05$; compare with the Lotensin group, ${ }^{\wedge} P<0.05$; compare between TCM groups, ${ }^{\star} P<0.05$.

\section{The Comparison of Urine Protein Quantitative Change in 24h Between Groups of Rats before and After Treatment}

Compared with model group, every groups'24h UTP decreased and TCM medium dose group decreased extraordinary $(P<0.05)$; As shows in table 2 .

Table 2 the comparison of $24 \mathrm{~h}$ UTP between groups of rats before and after treatment $(\mathrm{mg} / 24 \mathrm{~h})$

\begin{tabular}{ccccccc}
\hline $\begin{array}{c}\text { we } \\
\text { ek }\end{array}$ & $\begin{array}{c}\text { the blank } \\
\text { group }\end{array}$ & the model group & $\begin{array}{c}\text { the Lotensin } \\
\text { group }\end{array}$ & $\begin{array}{c}\text { the high dose } \\
\text { group }\end{array}$ & $\begin{array}{c}\text { the medium dose } \\
\text { group }\end{array}$ & $\begin{array}{c}\text { the low dose } \\
\text { group }\end{array}$ \\
\hline 0 & $25.65 \pm 0.56$ & $32.21 \pm 0.45$ & $32.11 \pm 0.44$ & $32.52 \pm 0.43$ & $43.26 \pm 0.73$ & $46.55 \pm 0.36$ \\
4 & $43.23 \pm 0.39$ & $77.15 \pm 0.71$ & $82.83 \pm 0.46$ & $69.85 \pm 0.34$ & $76.56 \pm 0.23$ & $80.26 \pm 0.22$ \\
6 & $49.87 \pm 0.52$ & $122.15 \pm 0.99^{\#}$ & $130.55 \pm 0.22^{\#}$ & $136.56 \pm 0.68^{\#}$ & $135.80 \pm 0.43^{\#}$ & $146.69 \pm 0.66^{\#}$ \\
8 & $48.64 \pm 0.31$ & $626.30 \pm 15.24^{\# \#}$ & $632.30 \pm 0.48^{\# \#}$ & $660.50 \pm 0.22^{\# \#}$ & $659.51 \pm 0.57^{\# \#}$ & $645.30 \pm 0.36^{\# \#}$ \\
10 & $40.70 \pm 1.24$ & $772.13 \pm 1.16$ & $626.85 \pm 1.10$ & $610.82 \pm 1.32$ & $542.71 \pm 1.19$ & $564.54 \pm 1.49$ \\
12 & $46.19 \pm 1.17$ & $978.69 \pm 1.63$ & $589.30 \pm 1.20$ & $527.48 \pm 1.71^{\triangle \Delta}$ & $410.71 \pm 1.86^{\Delta \Lambda}$ & $512.25 \pm 1.73^{\triangle \Delta}$ \\
14 & $46.34 \pm 1.65$ & $938.23 \pm 1.41$ & $516.61 \pm 1.50$ & $454.63 \pm 1.62^{\triangle}$ & $406.19 \pm 2.02^{\triangle}$ & $461.53 \pm 1.58^{\Delta}$ \\
16 & $48.01 \pm 0.68$ & $987.85 \pm 1.15$ & $431.21 \pm 0.62$ & $370.10 \pm 0.52^{\triangle}$ & $317.90 \pm 0.98^{\triangle \Delta}$ & $399.16 \pm 0.63^{\triangle \Delta}$ \\
\hline
\end{tabular}

Note: compare with the blank group ${ }^{\#} P<0.05,{ }^{\#} P<0.01$.compare with the model group, ${ }^{\Delta} P<0.05$; compare with Lotensin group, ${ }^{\wedge} P<0.05$; compare between TCM groups, $P<0.05$

\section{The Comparison of the Content of TNF-aand IL-6 in Rats Serum after Treatment between Groups of Rats}

Compared with blank group, TNF- $\alpha$, IL- 6 of model group's were significantly elevated $(P<0.05)$; compared with model group, TNF- $\alpha$, IL-6 of treatment groups decreased significantly $(P<0.05)$, compared between TCM group,TCM medium dose group's data is the most significant $(P<0.01)$. As shows in table 3. 
Table 3 the comparison of TNF- $\alpha$ and IL-6 in rats serum after treatment between groups of $\operatorname{rats}(\mathrm{pg} / \mathrm{ml})$

\begin{tabular}{|c|c|c|c|}
\hline group & $\mathrm{n}$ & TNF $-\alpha$ & IL-6 \\
\hline the blank group & 8 & $1.99 \pm 0.12$ & $1.24 \pm 0.12$ \\
\hline the model group & 13 & $3.79 \pm 0.47^{a}$ & $3.32 \pm 0.63^{H}$ \\
\hline the Lotensin group & 10 & $2.63 \pm 0.78^{\Delta}$ & $1.96 \pm 0.74^{\Delta}$ \\
\hline the high dose group & 10 & $2.01 \pm 1.05^{\Delta \boldsymbol{\Lambda}}$ & $1.16 \pm 0.63^{\Delta \Delta}$ \\
\hline the medium dose group & 10 & $1.90 \pm 0.52^{\Delta \Lambda \bullet}$ & $0.98 \pm 0.52^{\Delta \Lambda \bullet}$ \\
\hline the low dose group & 10 & $1.95 \pm 0.76^{\Delta \Delta}$ & $1.05 \pm 0.85^{\Delta \Lambda}$ \\
\hline
\end{tabular}

Note: compare with the blank group ${ }^{\#} P<0.05$, compare with the model group, ${ }^{\Delta} P<0.05$; compare with Lotensin group, ${ }^{\wedge} P<0.05$; compare between TCM groups, $P<0.05$

\section{Conclusion}

National physician master and professor Jixue Ren says that the core pathogenesis of the disease is kidney and spleen deficient, stagnation of pathogen in throat is causative factors of the disease. The throat can contact lung through renal, for the virus enter the body from nose and mouth, and entrench in the throat, which may violate the renal, forming a vicious cycle of throat, lung and kidney[4-7].Therefore nephritis occurrence and recurrence and the deterioration of the illness are closely related to the throat[8-10]. So he came up with tonifying kidney and spleen, detoxification of throat method, and it has significant curative effects in clinical application for more than 40 years [11-13]. Radix rehmanniae, radix astragali, Dangshen are important drugs for tonifying kidney and spleen; Golden buckwheat belongs to cool and bitter plan, but it is good at heat-clearing and detoxicating, and it is a good choice for treating swelling and sore throat [14]. Wood butterfly and puffball have function of detoxification of throat,and can cure cough, lung hot and voiceless. Cortex cercis and turmeric are good at resolving toxin and dispersing swelling; Smilax glabra is useful to resolve toxin and remove moisture and the urine protein; Lalang grass rhizome is good at reducing fever and causing diuresis, cooling blood and hemostasis. All of these drugs can take blood detoxification and tonify kidney.

The experimental results show that the Chinese traditional medicine group can obviously reduce the urine red blood cell count and 24h UTP, significantly decreased the content of TNF- $\alpha$ and IL-6 in serum. We thinks the tonifying kidney and spleen, detoxification of throat method can relief the deterioration of renal function by reducing the content of TNF- $\boldsymbol{\alpha}$ and IL-6 in serum which will inhabit the proliferation of glomerular mesangial cells and extracellular matrix production.

\section{Acknowledgement}

This research was financially supported by project as follows: Jilin Province Science and Technology Department of Natural Science Fund Project (No. 2015101214JC).

Jilin Province Administration of traditional Chinese medicine science and technology project (No. 2014-zd7)

Jilin Province Education Department "Twelfth Five Year Plan" science and technology research project (No. 65 of 2014). 


\section{Reference}

[1] Wang jun, Cheng xiaoxia, Zhu xiaoling, etc. the influence of the compound centella mixture to kidney disease in the rat kidney tissue inflammation factors and the expression [J](in Chinese). Chinese Journal of Integrated Traditional and Western Nephrology, 2008, 9 (11) : 949-952.

[2] Wang haiyan. Nephrology[M]3 edition(in Chinese). Beijing: People's Medical Publishing House, 2008:993.

[3] Liu Huiyu, Zhang qiaoling, Jiang xiaoyun, etc. IgAN rats model [J] (in Chinese) The misdiagnosis, 2011, 11 (6) : 1264-1267.

[4] Huang guodong, Wen huitao Ma xiaolu, etc. The influence of compound bone capsule to IL - 6 and TNF- $\alpha$ of nephrotic rats kidney tissue[J] (in Chinese). Journal of liaoning university of traditional Chinese medicine, 2009, 11 (11) : 2009-208.

[5] Wang jinnian.The clinical significance of detecting patients with chronic nephritis of $1 \mathrm{~L}-2,1 \mathrm{~L}-6$ and TNF- $\alpha$ in serum before and after treatment in patients [J] (in Chinese). Radiation immunology journal, 2008, 21 (5) : 394.

[6] Lin jian.The influence of tripterygium Glycosides to the contenct of $\operatorname{IgA}$ nephropathy model $\mathrm{IL}-2,1 \mathrm{~L}-6$ and TNF-a in rats serum[J] (in Chinese). Chinese and foreign medical, 2011 (4) : 21-22.

[7] Liu xiaoping, Hu jinshun, Fang yi. The establishment of rats IgA nephropathy model and the detection of IL - 6, FN and NO in serum [J] (in Chinese). Chinese journal of comparative medicine, 2008, 19 (1) : 5-8.

[8] Ruef C.Interleukin-6 is an autscrine growth factor for mesang-ial cells[J].Kideney Int, 1990,38: 249.

[9] Shi xiuyan $\mathrm{Hu}$ zhaoxiong, Zhu zhonghua. The plasma of patients with chronic nephritis and the clinical significance of serum Leptin and serum IL - 6, IL - 18 and SOD [J] (in Chinese). The immune radiology journal, 2012, 25 (2) : 125-127.

[10] Ye rengao, Zeng lixia. Patients with mesangial cells hyperplastic glomerular nephritis IL - 6, IL - 18 and SOD expression and its significance [J] (in Chinese). the China' kidney disease, 1998, 14 (1) : 3-6.

[11]Liu yanhua, Ren xijie, Wang jian, etl. Professor Ren Jixue application laryngeal chronic kidney related theory experience of diagnosis and treatment of renal[J] (in Chinese). Journal of traditional Chinese medicine, 2015 46-48 (4) : 283-285.

[12] Tian yudong, Ma defeng, Ren xijie. Professor Ren Jixue experience of treating kidney wind exogenous medicine $[\mathrm{J}]$ (in Chinese). Chinese community physicians, 2014, 30 (1) : 93-95.

[13] Yang li. Professor Ren Jixue TCM theory on glomerulonephritis [J] (in Chinese). Journal of guangzhou university of Chinese medicine, 2003, 20 (1): 79-81.

[14] Tong yanqing. Ren jixue experience of treating chronic renal failure medicine [J] (in Chinese).Chinese medicine literature journal, 2004(1):34-36. 